\title{
A New Application Using a Chromogenic Assay in a Plant Pathogen DNA Macroarray Detection System
}

Mui-Yun Wong, Department of Plant Protection, Faculty of Agriculture, and Institute of Tropical Agriculture, Universiti Putra Malaysia, Serdang, Selangor 43400, Malaysia; and Christine D. Smart, Department of Plant Pathology and Plant-Microbe Biology, Cornell University, Geneva, NY 14456

\begin{abstract}
Wong, M.-Y., and Smart, C. D. 2012. A new application using a chromogenic assay in a plant pathogen DNA macroarray detection system. Plant Dis. $96: 1365-1371$.

A DNA macroarray was previously developed to detect major fungal and oomycete pathogens of solanaceous crops. To provide a convenient alternative for researchers with no access to X-ray film-developing facilities, specific CCD cameras or Chemidoc XRS systems, a chromogenic detection method with sensitivity comparable with chemiluminescent detection, has been developed. A fungal (Stemphylium solani) and an oomycete (Phytophthora capsici) pathogen were used to develop the protocol using digoxigenin (DIG)-labeled targets. The internal transcribed spacer (ITS) region of the nuclear ribosomal DNA (rDNA), including ITS1, 5.8S rDNA, and ITS2, was used as the target gene and polymerase chain reaction amplified as in the previous protocol. Various amounts of species-specific oligonucleotides on the array, quantities of DIG-labeled ITS amplicon, and hybridization temperatures were tested. The optimal conditions for hybridization were $55^{\circ} \mathrm{C}$

for $2 \mathrm{~h}$ using at least $10 \mathrm{pmol}$ of each species-specific oligonucleotide and labeled target at $10 \mathrm{ng} / \mathrm{ml}$ of hybridization buffer. Incubation of the hybridized array with anti-DIG conjugated alkaline phosphatase substrates, NBT/BCIP, produced visible target signals between 1 and $3 \mathrm{~h}$ compared with $1 \mathrm{~h}$ in chemiluminescent detection. Samples from pure cultures, soil, and artificially inoculated plants were also used to compare the detection using chemiluminescent and chromogenic methods. Chromogenic detection was shown to yield similar results compared with chemiluminescent detection in regard to signal specificity, duration of hybridization between the array and targets, and cost, though it takes 1 to $2 \mathrm{~h}$ longer for the visualization process, thus providing a convenient alternative for researchers who lack darkroom facilities. To our knowledge, this is the first report of DNA macroarray detection of plant pathogens using a chromogenic method.
\end{abstract}

In plant disease management, rapid and accurate detection and identification of pathogens is essential before any effective control measures can be recommended. Disease diagnosis using conventional methods such as culturing and morphological characterization is tedious and time consuming and requires extensive taxonomical knowledge. Alternative methods are needed which provide high throughput and rapid results. The development of DNA array technology (11) has provided a useful and powerful tool that could overcome difficulties in pathogen identification encountered when using conventional methods. DNA array technology is essentially a reverse dot-blot technique which has been used in various applications, including the detection of human genetic disorders $(4,11)$, environmental and ecological studies (14), detection of foodborne pathogens $(2,9)$, and detection of plant pathogens $(1,3,5,6,13,16,17)$. Using this technology, multiple pathogens can be detected simultaneously to species and intraspecies levels with highly specific detector oligonucleotides $(8,16,17)$.

Macroarrays are flexible membrane-based oligonucleotide arrays which generally contain hundreds of species-specific probes. In contrast to microarrays, this detection method is more sensitive because it uses higher concentrations of DNA probes. It is also more cost effective because it utilizes inexpensive reagents, and the array can reliably be reused many times. The results are highly reproducible; thus, its application is suitable for high-throughput

Corresponding author: M.-Y. Wong, E-mail: muiyun@putra.upm.edu.my

Accepted for publication 17 March 2012.

http://dx.doi.org/10.1094/PDIS-07-11-0593-SR

(C) 2012 The American Phytopathological Society screening of samples of diseased plants, soil, and water $(14,17)$. The internal transcribed spacer (ITS) region within the nuclear ribosomal DNA (rDNA) gene is the most commonly used target for identification of eukaryotic pathogen species due to the variability in the ITS sequences, which enables discrimination of closely related species even with a single nucleotide difference $(8,12,16,17)$ and the large number of rDNA sequences available in the public domain.

A DNA macroarray detection system consists of five steps: (i) development of a species-specific array, (ii) extraction of DNA from samples, (iii) polymerase chain reaction (PCR) amplification and labeling of targets, (iv) hybridization of labeled targets with the array, and ( $\mathrm{v}$ ) detection of signals produced by hybridized detector oligonucleotides. Signals can be detected using several methods, including chemiluminescence and chromogenesis. Chemiluminescent detection is based on the emission of light from the enzyme activity of alkaline phosphatase on its substrate while chromogenic detection is based on the formation of dark blue or indigo insoluble precipitates through the same enzyme activity (10).

A vegetable pathogen macroarray developed previously in our laboratory to detect major fungal and oomycete pathogens of solanaceous crops using chemiluminescent detection proved to be reliable and efficient (17). This array contains 105 oligonucleotides with the ability to detect and differentiate 25 fungal and oomycete pathogens plus 12 members of the Fusarium solani species complex, with each set of species-specific oligonucleotides spotted as quadruplets in the array and arranged in either an " $L$ " or "inverted-L" pattern. However, the darkroom facility requirement of this method may be a limitation for many researchers. In contrast, chromogenic detection can be carried out at the workbench at any time. Therefore, the objective of this work was 
to develop an optimized protocol for a chromogenic detection method and compare it with chemiluminescent detection.

\section{Materials and Methods}

Fungal isolates. The fungus Stemphylium solani (American Type Culture Collection 11128) and oomycete Phytophthora capsici (0664-1) were used in this study. The pure cultures were maintained following the protocol of Zhang et al. (17).

Greenhouse and field samples. Four-week-old Capsicum annuum 'Red Knight' plants were inoculated with $10^{5}$ zoospores of $P$. capsici (0664-1) and maintained in the greenhouse for 7 days. For field samples, six soil samples were taken from two high tunnels planted with cherry tomato from Livonia, NY. The tomato plants died during the previous season but the cause of death was unknown. Each soil sample was taken with a core for a depth of approximately $10-15 \mathrm{~cm}$, kept in a plastic bag, and stored at $4^{\circ} \mathrm{C}$ until use.

Genomic DNA extraction. Genomic DNA extraction from pure cultures of each pathogen and from diseased plants was carried out using the UltraClean Plant DNA Isolation Kit (MoBio Laboratories). All six soil samples from the field were air dried and $1 \mathrm{~g}$ of each sample was mixed well manually to produce a representative sample before performing genomic DNA extraction following the protocol of the PowerSoil DNA Isolation Kit (MoBio Laboratories). Extracted DNA was stored at $-20^{\circ} \mathrm{C}$ until use.

Target amplification, labeling, and purification. The ITS regions of target pathogens were amplified using ITS universal primers ITS4 and ITS5 (15). The target was simultaneously labeled with digoxigenin (DIG) during PCR amplification following the manufacturer's protocol using the PCR DIG Labeling Mix (Roche). It is important to note that the labeling reaction used is identical preceding both chemiluminescent and chromogenic detection. The labeling method used in this study was different from that in Zhang et al. (17) because the latter used Gene Images AlkPhos Direct Labeling system (Amersham Biosciences). Each 50- $\mu$ PCR reaction consisted of $1 \times$ PCR buffer plus $1.5 \mathrm{mM}$ $\mathrm{MgCl}_{2} ; 1 \times$ PCR DIG labeling mix, including $200 \mu \mathrm{M}$ final concentration of dNTPs; $0.2 \mu \mathrm{M}$ each primer; 1 unit of Taq DNA polymerase; and 14 to $25 \mathrm{ng}$ of genomic DNA. PCR was run using the protocol of Zhang et. al. (17): $95^{\circ} \mathrm{C}$ for $5 \mathrm{~min}$; 35 cycles of $95^{\circ} \mathrm{C}$ for $1 \mathrm{~min}, 56^{\circ} \mathrm{C}$ for $1 \mathrm{~min}$, and $72^{\circ} \mathrm{C}$ for $1 \mathrm{~min}$; followed by 10 min at $72^{\circ} \mathrm{C}$. PCR products were purified using the UltraClean PCR Clean-Up Kit (MoBio Laboratories) according to the manufacturer's protocol.

Detector oligonucleotide array production. To test the optimal hybridization temperature, a vegetable pathogen array was pro-

Table 1. Optimization of hybridization temperature for chemiluminescent and chromogenic detection ${ }^{\mathrm{a}}$

\begin{tabular}{llccc}
\hline Oligo name & Description or target & $\mathbf{3 7}^{\circ} \mathbf{C}$ & $\mathbf{4 2}^{\circ} \mathbf{C}$ & $\mathbf{5 5}^{\circ} \mathbf{C}$ \\
\hline ITS2 & Positive control & - & - & + \\
ITS4 & Positive control & - & - & + \\
ITS5 & Positive control & - & - & + \\
ITS2_1_1 & Internal control & - & - & - \\
ITS2_1_2 & Internal control & - & - & - \\
ITS2_1_3 & Internal control & - & - & + \\
ITS2_2_1 & Internal control & - & - & - \\
ITS2_2_2 & Internal control & - & - & - \\
Sts1 & Stemphylium solani & - & - & + \\
Sts2 & S. solani & - & - & + \\
Sts3 & S. solani & - & - & + \\
Sts4 & S. solani & + & + & + \\
Pc2 & Phytophthora capcisi & - & - & + \\
Pc3 & P. capcisi & - & - & + \\
Pc4 & P. capcisi & - & - & + \\
Pc6 & P. capcisi & - & - & + \\
Water & Negative control & - & - & - \\
\hline
\end{tabular}

a Species-specific oligonucleotides (10 pmol each) were incubated with targets at $10 \mathrm{ng} / \mathrm{ml}$ of hybridization buffer overnight; $+=$ visible hybridization signal and $-=$ no visible hybridization signal. Results were identical for both chemiluminescent and chromogenic detection. ITS = internal transcribed spacer. duced according to the protocol of Zhang et. al. (17) using $30 \mathrm{pmol}$ of species-specific oligonucleotides instead of $10 \mathrm{pmol}$. Zhang et al. (17) reported using $10 \mathrm{fmol}$ of each detector oligonucleotide for spotting onto the nylon membranes, which was an error (as confirmed by N. Zhang). To test the optimal concentration of speciesspecific oligonucleotides and quantity of DIG-labeled amplicons needed for chromogenic detection, an array consisting of 10, 20, 30, 40, and 50 pmol of species-specific oligonucleotides each for $S$. solani and $P$. capsici was developed. Probes used for $S$. solani and $P$. capsici detection were Sts1, Sts2, Sts3, and Sts4 and Pc2, Pc3, Pc4, and Pc6, respectively, which were developed previously in our laboratory (17). Positive controls used were one fungal and oomycete universal primer (ITS4) and one fungal universal primer (ITS2) (17). Sterile water was used as negative control. Internal controls were not used due to the fact that the specificity of the species-specific oligonucleotides used in this study was verified by Zhang et al. (17) inclusive of internal controls. The oligonucleotides were spotted onto nylon membranes (GE Healthcare) using a 96-pin replicator (model 250520; Nalge Nunc International Corp.) in quadruplicate. After spotting, membranes were air dried for at least $10 \mathrm{~min}$ and fixed by UV exposure at $240 \mathrm{~mJ} / \mathrm{cm}^{2}$. Membranes were stripped using $0.5 \%$ sodium dodecyl sulfate (SDS) at $60^{\circ} \mathrm{C}$ for $1 \mathrm{~h}$, washed in $100 \mathrm{mM}$ Tris ( $\mathrm{pH} \mathrm{8.0)}$ ) for $5 \mathrm{~min}$, and kept moist at $4^{\circ} \mathrm{C}$ until use.

Hybridization and detection. To test the optimal hybridization temperature, the vegetable pathogen array and DNA from pure cultures of $S$. solani and $P$. capsici were used. Hybridization of DIG-labeled amplicons and species-specific arrays using DIG Easy Hyb (Roche) solution was conducted according to the manufacturer's protocol with slight modifications. DIG Easy Hyb (20 ml) was preheated and incubated with each vegetable pathogen arrayspotted membrane in hybridization tubes for $30 \mathrm{~min}$. DIG-labeled amplicons (targets at $10 \mathrm{ng} / \mathrm{ml}$ of hybridization buffer) were denatured at $98^{\circ} \mathrm{C}$ for $5 \mathrm{~min}$ in a thermal cycler (Model Mastercycle Gradient; Eppendorf) instead of boiling for 5 min according to the manufacturer, then immediately cooled on ice. After 5 min of cooling, the amplicons were added to $20 \mathrm{ml}$ of preheated DIG Easy Hyb. After $30 \mathrm{~min}$, the prehybridization solution was poured off the hybridization tubes, the preheated DIG Easy Hyb containing DIG-labeled amplicons was immediately added, and the tubes were incubated overnight at 37,42 , and $55^{\circ} \mathrm{C}$ separately. This hybridization step was repeated at least twice for each temperature tested and each detection method. These temperatures were selected based on the recommendations by the manufacturer (Roche) and the previous protocol (17). After hybridization, the membranes were washed with $2 \times \mathrm{SSC}(1 \times \mathrm{SSC}$ is $0.15 \mathrm{M} \mathrm{NaCl}$ plus $0.015 \mathrm{M}$ sodium citrate) containing $0.1 \%$ SDS for $5 \mathrm{~min}$ twice at room temperature, and $1 \times$ SSC containing $0.1 \%$ SDS for $15 \mathrm{~min}$ twice at hybridization temperature. For chemiluminescent detection, CDPStar (Amersham Biosciences) was added to the membranes to react for $1 \mathrm{~h}$ followed by $30 \mathrm{~min}$ of exposure on Kodak Biomax Light films (Sigma-Aldrich) in cassettes. The films were developed and hybridization signals determined by scanning the films with an HP ScanJet scanner (model 5300C) and saving the scans as a softcopy. For chromogenic detection, the membranes were incubated with anti-DIG antibody conjugated with alkaline phosphatase and subsequently with NBT/BCIP substrates (Roche) according to the protocol of the manufacturer. Hybridization signals were determined by taking photographs of the membranes under white fluorescence light in a standard gel imaging device and saving the images as softcopies. Comparison of hybridization signal intensity using gray value was not possible due to the different methods used for capturing signals between chemiluminescent and chromogenic detections as mentioned above. Therefore, hybridization signal differences between the two detection methods were assessed by subjectively comparing the visible patterns of hybridization.

Used membranes were stripped for reprobing following either chemiluminescent or chromogenic detection. Membranes used in chemiluminescent detection were stripped according to Zhang et 
al. (17). Membranes used in chromogenic detection were stripped according to the protocol of the DIG Nucleic Acid Detection Kit (Roche). Briefly, membranes were incubated in heated (50 to $60^{\circ} \mathrm{C}$ ) dimethylformamide for $20 \mathrm{~min}$ or until the blue color precipitate was removed from the membranes. The membranes were then rinsed briefly with double-distilled water before washing twice for $20 \mathrm{~min}$ in $0.2 \mathrm{~N} \mathrm{NaOH}$ and $0.1 \% \mathrm{SDS}$ (wt/vol) at $37^{\circ} \mathrm{C}$ under constant agitation. After equilibrating the membranes briefly in $2 \times \mathrm{SSC}$ buffer, they were air dried and kept at $4^{\circ} \mathrm{C}$ or used immediately.

For testing the optimal amount of species-specific oligos on the array and quantity of DIG-labeled amplicons needed for chromogenic detection, the optimum hybridization temperature obtained from the test above was used for both pathogens using pure cultures. For each species, two identical sets of array-spotted membrane were hybridized with DIG-labeled ITS amplicons using concentrations of labeled amplicon at 5, 10, and $15 \mathrm{ng} / \mathrm{ml}$ of DIG Easy Hyb buffer overnight. One array-spotted membrane was used for chemiluminescent detection and a second membrane for chromogenic detection for each species. Membrane washing and chemiluminescent and chromogenic detection were conducted as described above. In addition to overnight hybridization, hybridization of the array and targets for the duration of $2 \mathrm{~h}$ was also tested. Optimized conditions were determined by comparing the visible pattern of hybridization specificity. All hybridization tests were repeated at least twice with amplicons produced in separate PCR reactions.

For soil and plant samples, hybridization of the vegetable pathogen array and DIG-labeled ITS amplicons was conducted using the optimal hybridization temperature, amount of species-specific oligos on the array, quantity of DIG-labeled amplicons, and hybridization duration obtained from tests mentioned above.

\section{Results}

Using the vegetable pathogen array containing 10 pmol of species-specific oligonucleotides and $10 \mathrm{ng}$ of labeled target $/ \mathrm{ml}$ hybridization buffer, the hybridization temperatures of 37,42 , and $55^{\circ} \mathrm{C}$ were tested and signals were detected using the chromogenic method. The results were comparable with chemiluminescent detection. At hybridization temperatures of 37 and $42^{\circ} \mathrm{C}$, visible signals were produced only from $S$. solani-specific oligonucleotides and none from $P$. capsici-specific oligonucleotides (Table 1). At a hybridization temperature of $55^{\circ} \mathrm{C}$, signals from both speciesspecific oligonucleotides were visible (Table 1). Therefore, the latter temperature was used in subsequent tests using $S$. solani and $P$. capsici-specific arrays.

Species-specific arrays for $S$. solani and $P$. capsici were designed and spotted to determine the minimal concentration of oligonucleotide and labeled target amplicon required to detect visible signals using chromogenic compared with chemiluminescent detection. Visible signals from species-specific oligonucleotides and positive controls were detected when labeled target amplicon of at least $10 \mathrm{ng} / \mathrm{ml}$ of hybridization buffer was used in a hybridization with at least $10 \mathrm{pmol}$ of oligonucleotide on the array for both microorganisms tested (Figs. 1 and 2).

For the $S$. solani-specific array, visible patterns of hybridization using both chemiluminescent and chromogenic methods were similar. Hybridization of labeled target at $5 \mathrm{ng} / \mathrm{ml}$ of hybridization buffer and 10 to $50 \mathrm{pmol}$ of specific oligonucleotides produced visible signals for Sts2 and Sts4 only (Fig. 1A and B). Signals for
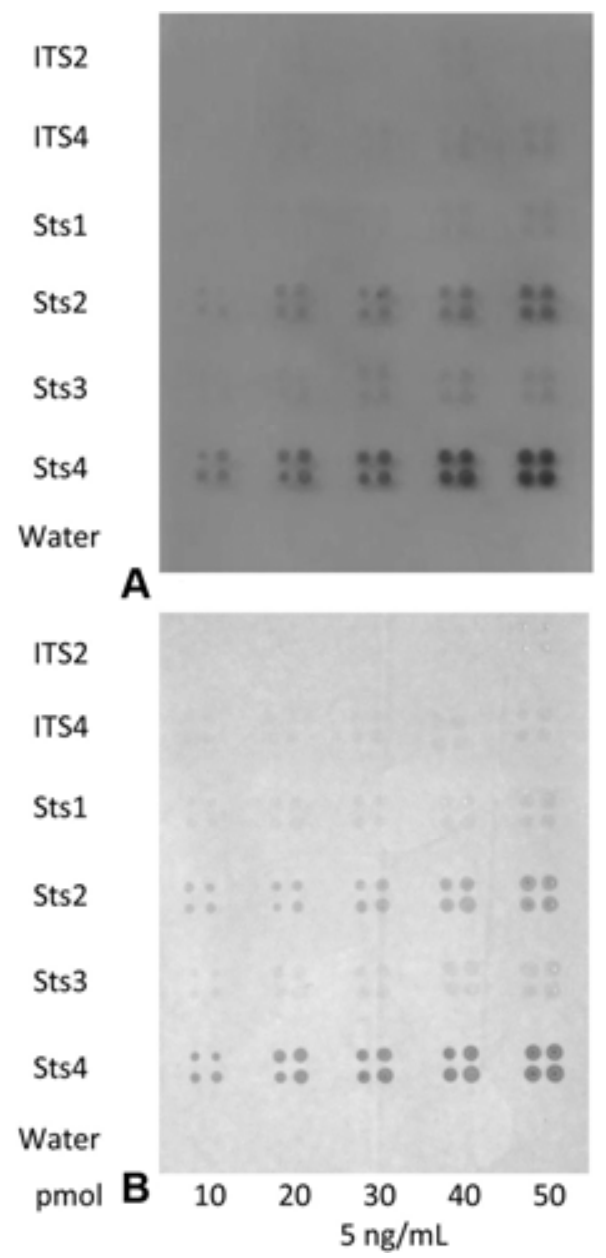

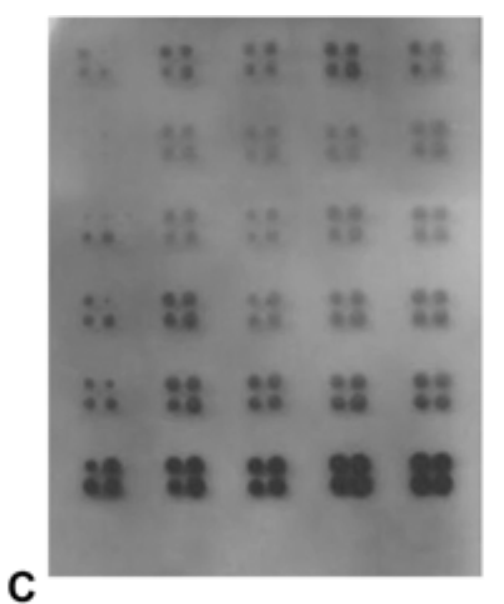

C

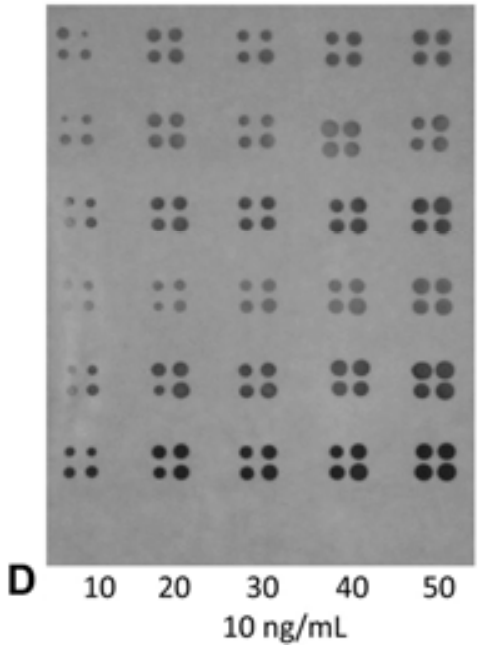

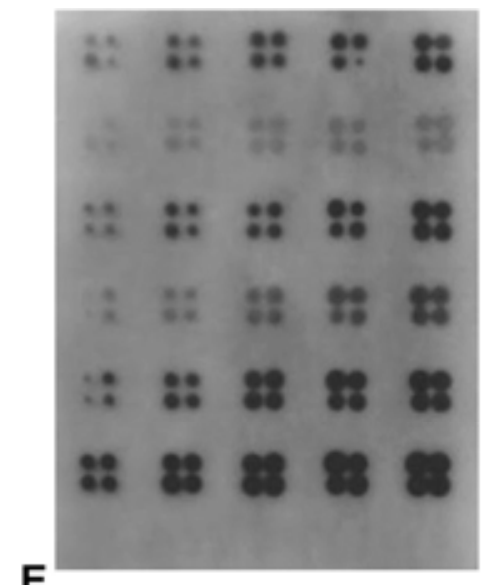

E

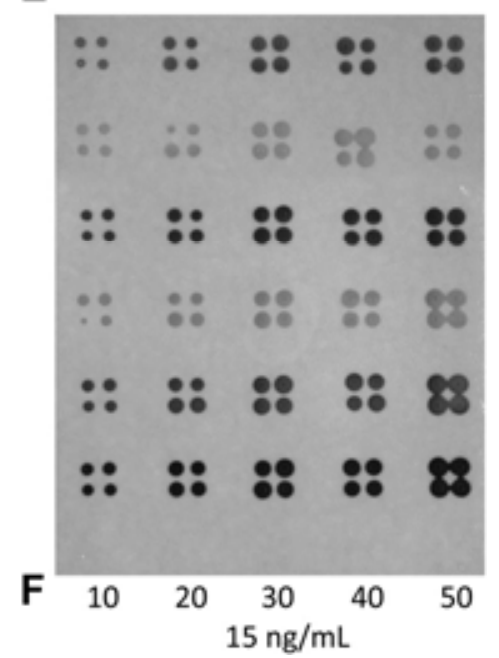

Fig. 1. Effect of oligonucleotide and target concentration on signal production using a Stemphylium solani-specific array. Detection using chemiluminescent (A, C, and E) and chromogenic (B, D, and F) methods after overnight hybridization at $55^{\circ} \mathrm{C}$. Internal transcribed spacer (ITS)2 and ITS4, positive controls; Sts1 to -4 , species-specific oligonucleotides; and Water, negative control. 
ITS2, ITS4, Sts1, and Sts4 were not clearly visible using both detection methods. There was no signal detected for the negative control (water). When the amount of labeled target was increased to $10 \mathrm{ng} / \mathrm{ml}$ of hybridization buffer, hybridization signal patterns for Sts 1 to -4 produced weak but visible signals when spotted at 10 pmol compared with 20,30, 40, and 50 pmol, which produced intense signals using both detection methods (Fig. 1C and D). Signals from ITS2 and ITS4 were also visible using at least $10 \mathrm{pmol}$ of specific oligonucleotides for hybridization. No signal was visible for the negative control (water). Hybridization using the labeled target at $15 \mathrm{ng} / \mathrm{ml}$ of hybridization buffer and 10 to $50 \mathrm{pmol}$ of each specific oligonucleotide produced visible signal patterns similar to using labeled target at $10 \mathrm{ng} / \mathrm{ml}$ of hybridization buffer (Fig. $1 \mathrm{E}$ and $\mathrm{F})$.

For the $P$. capsici-specific array, hybridization of labeled target at $5 \mathrm{ng} / \mathrm{ml}$ of hybridization buffer and 10 to $50 \mathrm{pmol}$ of specific oligonucleotides produced either extremely weak or no visible signals from any oligonucleotides tested, including positive controls (ITS2 and ITS4), using both detection methods (Fig. 2A and B). Hybridization using labeled target at 10 and $15 \mathrm{ng} / \mathrm{ml}$ of hybridization buffer and 10 to $50 \mathrm{pmol}$ of specific oligonucleotides produced similar visible signal patterns for ITS4, Pc2, Pc3, and Pc6 using both detection methods (Fig. 2C-F). There was no signal detected for ITS2 or the negative control (water). However, a very weak hybridization signal was detected for ITS2 using labeled target at $15 \mathrm{ng} / \mathrm{ml}$ of hybridization buffer and more than $20 \mathrm{pmol}$ of oligonucleotides (Fig. 2E and F). For Pc4, the chemiluminescent method detected very weak but visible signals compared with none using chromogenic detection (Fig. 2E and F).
To determine the impact of reducing hybridization time on the specificity of the hybridization signal, the same species-specific arrays as above were used for hybridization with labeled targets at $10 \mathrm{ng} / \mathrm{ml}$ of hybridization buffer for a duration of $2 \mathrm{~h}$ using both chemiluminescent and chromogenic detection. For both the $S$. solani- and $P$. capsici-specific arrays, signal patterns using both methods were similar to those appearing after overnight hybridization (Figs. 3A and 1C, 3B and 1D, 3C and 2C, 3D and 2D).

Chromogenic detection produced visible signals within $2 \mathrm{~h}$ for $S$. solani and $3 \mathrm{~h}$ for $P$. capsici after incubation of the arrays and substrates (NBT/BCIP), and the intensity of signals did not increase after $6 \mathrm{~h}$ of incubation with substrates. The intensity of signals detected using both methods varied based on the individual oligonucleotides. For example, for $S$. solani, signals detected from Sts4 were the most intense compared with those detected from Sts1, Sts2, and Sts3; and, for P. capsici, signals detected from Pc2, Pc3, and Pc6 were much more intense than those detected from Pc4. This difference in signal detection from individual oligonucleotides was identical for both detection methods.

For detection from environmental samples, the solanaceous vegetable pathogen array was prepared using $30 \mathrm{pmol}$ of speciesspecific oligonucleotides and hybridized with labeled targets at 10 $\mathrm{ng} / \mathrm{ml}$ of hybridization buffer at $55^{\circ} \mathrm{C}$ for rapid detection based on the optimum conditions obtained from using pure cultures. The concentration of species-specific oligonucleotides selected was to ensure overall consistency in signal production by all the oligonucleotides spotted on the membrane even though 10 pmol was sufficient to produce visible detection of isolates in this study (Figs. 1 and 2). For the representative soil sample from the high

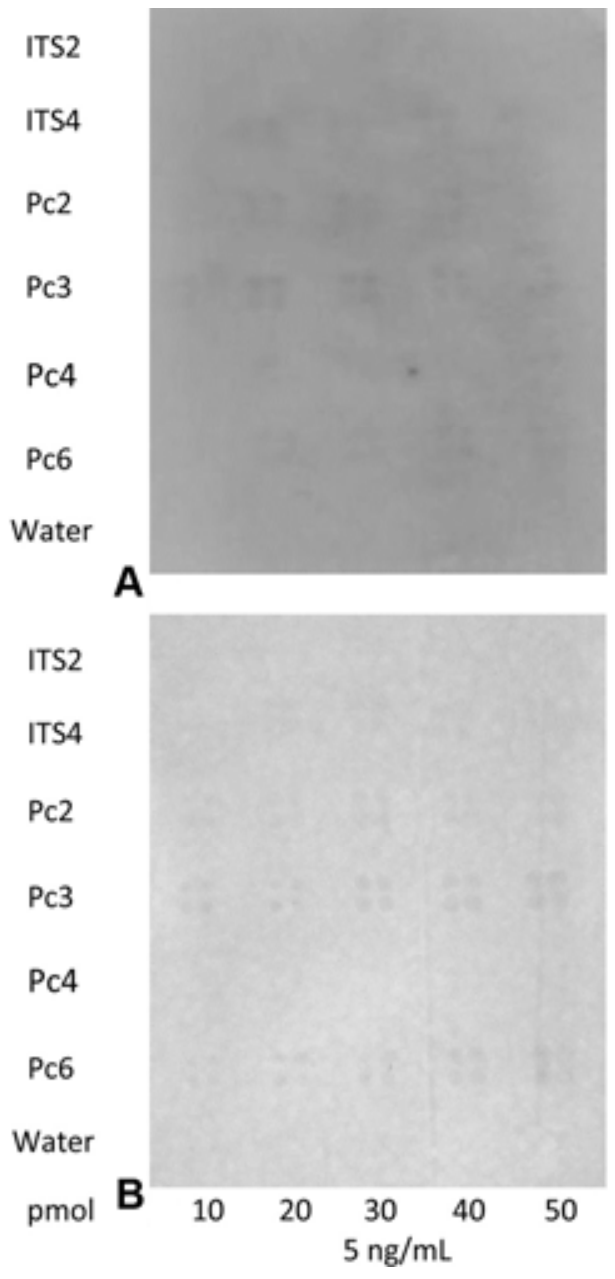

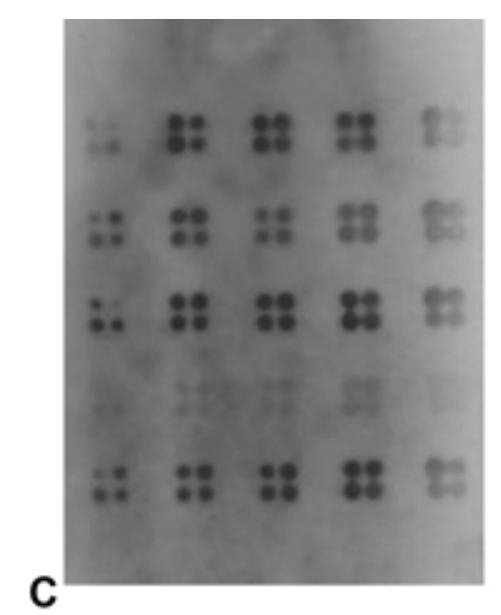
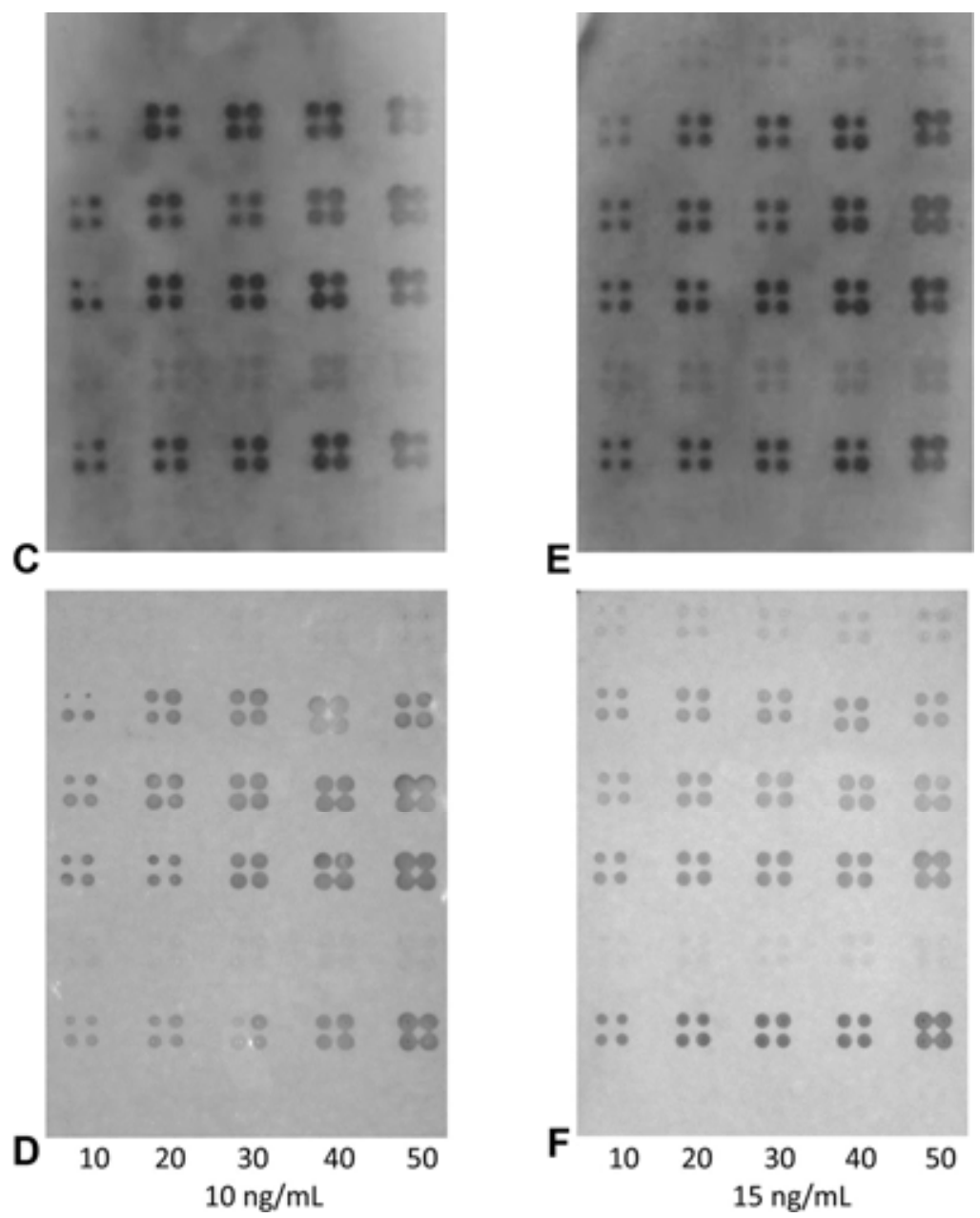

E

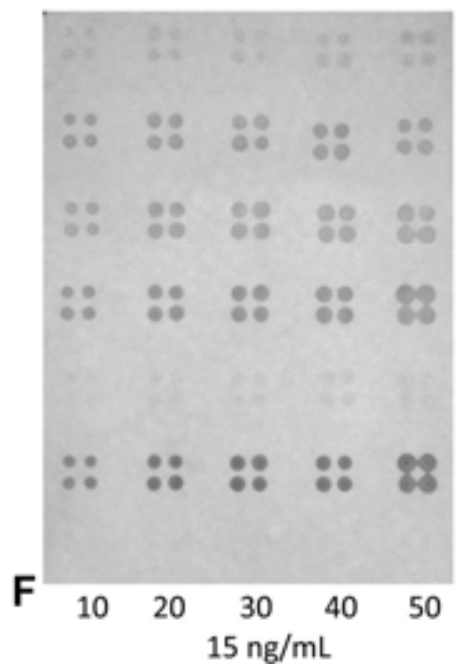

Fig. 2. Effect of oligonucleotide and target concentration on signal production using a Phytophthora capsici-specific array. Detection using chemiluminescent (A, C, and E) and chromogenic (B, D, and F) methods after overnight hybridization at 55 $\mathrm{C}$. Internal transcribed spacer (ITS)2 and ITS4, positive controls; Pc2, -3,- 4 , and -6, speciesspecific oligonucleotides; and Water, negative control. 
tunnels, signals from three pathogens were detected using both detection methods: Pyrenochaeta lycopersici, Phoma destructiva, and F. oxysporum (Fig. 4A and B). Meanwhile, signals from Phytophthora capsici and the solanaceous plant control oligonucleotides were detected in a pepper plant artificially inoculated with P. capsici (Fig. 4C and D). For the same sample (artificially inoculated pepper), signal intensity after $3 \mathrm{~h}$ of incubation of the vegetable pathogen array and chromogenic substrates was less for some oligonucleotides than those produced using chemiluminescent detection. However, after overnight incubation, the signal intensity increased and was similar to that of chemiluminescent detection (data not shown). Membranes with arrays used for either chromogenic or chemiluminescent detection were successfully stripped and reused at least 10 times during this study without compromising the intensity or specificity of the signal pattern.

\section{Discussion}

The application of DNA macroarray detection systems in plant disease diagnosis has become increasingly common due to the rapid and accurate detection and identification of multiple plant pathogens. Signals produced from the hybridization of labeled targets to species-specific oligonucleotides on the array can be detected using chemiluminescent or chromogenic detection meth- ods. Chemiluminescent detection is based on light emission during the reaction of alkaline phosphatase and its substrate, CDP-Star, and signals are captured on film. The film is developed in a darkroom facility, with specialized chemicals and equipment. Chromogenic detection utilizes signal from color precipitates produced during the reaction of the same enzyme with its substrates, NBTBCIP, in the dark. This detection method can be carried out conveniently on the workbench. In addition, membranes used for chromogenic detection were successfully reused multiple times, similar to that for chemiluminescent detection.

Results from this study show that patterns of hybridization signals detected using the chromogenic method are the same (Figs. 1 and 2) but slightly weaker (Fig. 4) though easily readable compared with that of chemiluminescent detection. In the authors' opinion, measurement of the intensity of signals produced by both detection methods is not critical because the chemiluminescent detection method is expected to be more sensitive than the chromogenic detection. Furthermore, the measurement for signal intensity using gray value was not possible due to different methods in capturing the signals between chemiluminescent and chromogenic detections, as mentioned above. What the authors want to show is that the specificity of hybridization signal patterns detected by each specific oligonucleotide using the chromogenic method is the same or similar to those detected using the chemiluminescent method.
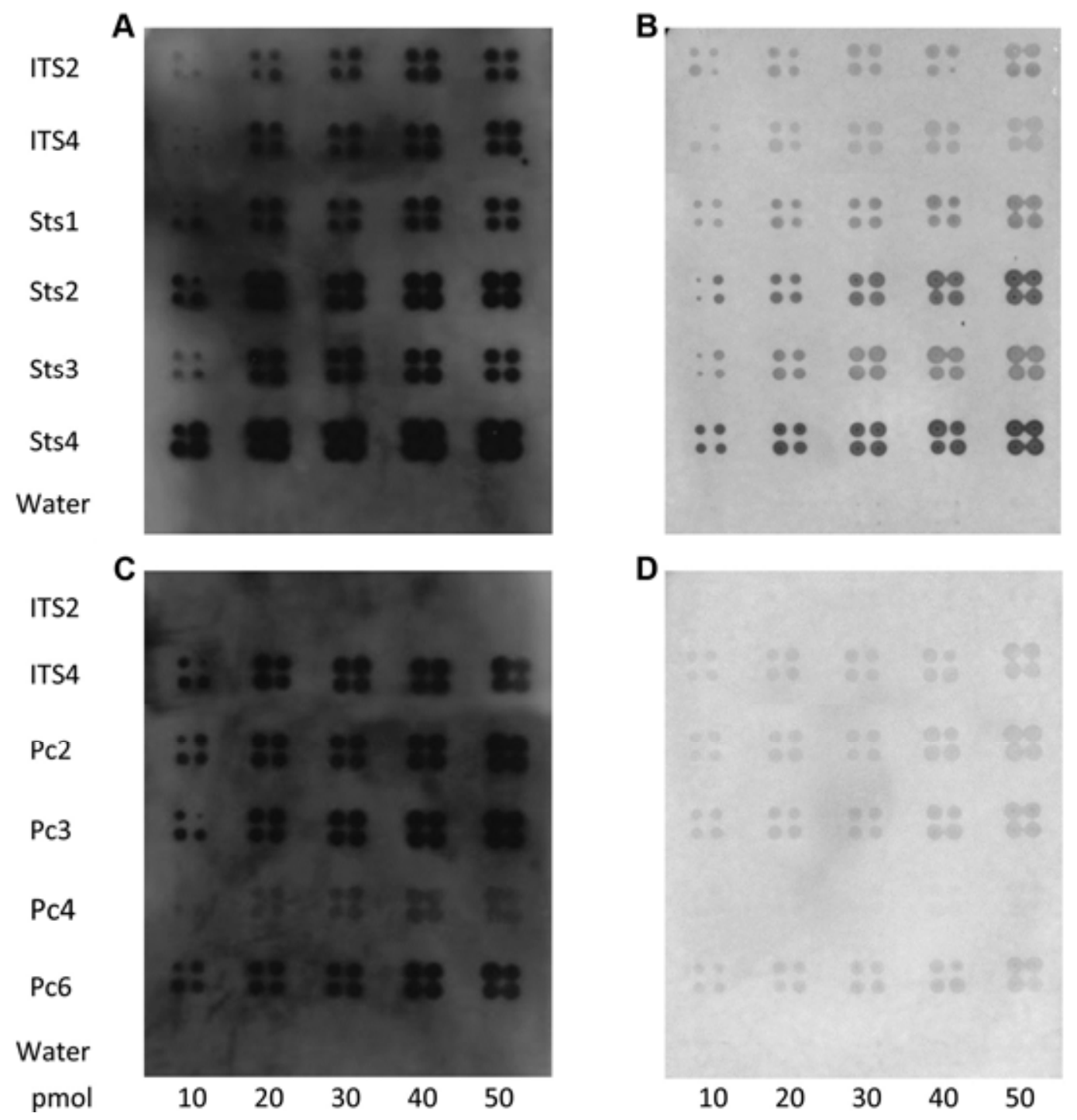

Fig. 3. Effect of oligonucleotide concentration on signal production at $2 \mathrm{~h}$ using Stemphylium solani- and Phytophthora capsici-specific arrays. Detection using chemiluminescent ( $\mathbf{A}$ and $\mathbf{C}$ ) and chromogenic (B and $\mathbf{D}$ ) methods after $2 \mathrm{~h}$ of hybridization with target at $10 \mathrm{ng} / \mathrm{ml}$ of hybridization buffer at $55^{\circ} \mathrm{C}$. Internal transcribed spacer (ITS)2 and ITS4, positive controls; Sts1 to -4 and Pc2, $-3,-4$, and -6, species-specific oligonucleotides; and Water, negative control. 
Furthermore, to detect a particular species, we used four specific oligonucleotides. If there was no signal detected by one oligonucleotide (for whatever reason), signals detected by three other oligonucleotides would confirm the specific detection of a particular species. In our study, the pattern of hybridization signals detected using the two detection methods showed similarity regardless of the type of sample used.

In another study related to food inspection, a chromogenic macroarray method was developed that provided convenient and simultaneous detection of the common enterotoxigenic Staphylococcus aureus strains which account for most food poisoning cases (9). The array was based on five major types of staphylococcal enterotoxins spotted on a nylon membrane and hybridized to biotin-labeled targets. This offers a low-cost alternative for average clinical and food inspection laboratories to perform detection of these strains in food samples. The relative hybridization signal strengths were determined by scanning the membranes with a UMAX device and comparing the specificity of the hybridization patterns of the oligonucleotide array.

In our study, a PCR-DIG labeling and detection system was used to label target DNA amplicons and detect hybridization signal using two methods (i.e., chemiluminescent and chromogenic detection). In this system, PCR products were labeled with DIG conjugated with alkaline phosphatase during amplification and, after hybridization, the arrays were incubated in blocking and anti-DIG antibody solutions and washed before they were incubated with substrates CDP-Star (for chemiluminescent detection) and NBTBCIP (for chromogenic detection). In terms of signal specificity, both detection methods produced similar results when speciesspecific arrays were used (Figs. 1 and 2). The specificity of the oligonucleotides for both $S$. solani and $P$. capsici detection was verified by Zhang et al. (17). Similar results were obtained when the vegetable array was used to detect soil and plant samples but the intensity of the chromogen was weaker than chemiluminescence (Fig. 4). In terms of processing time, the chemiluminescent system required slightly less time ( 1 to $2 \mathrm{~h}$ less) compared with the chromogenic system used in this study. This was due to the additional time needed to incubate the arrays in the antibody solution and the reaction time necessary for color precipitation. In each detection system, detection results were obtained within 1 day. In terms of cost, both detection methods are comparable. For a $100-\mathrm{cm}^{2}$ membrane, the cost per membrane for chemiluminescent detection was \$25.32 compared with \$26.79 for chromogenic detection (data not shown). However, if the washing and detection buffers are to be prepared in the laboratory, the cost for chromogenic detection will be further reduced.

The target labeling system used in this study was different from the one developed earlier in our laboratory (17), which used Gene Images AlkPhos Direct Labeling and Detection System with CDPStar. In the previous system, PCR targets were labeled with alkaline phosphatase after PCR amplification was completed. The results obtained from this study show that the PCR-DIG labeling system provides an alternative to the direct labeling system without compromising the detection results.

The effect of hybridization duration on signal production was also tested in this study. For both $S$. solani and $P$. capsici, signal specificity patterns detected after $2 \mathrm{~h}$ of hybridization of labeled targets and arrays using the chromogenic method were similar to those detected using the chemiluminescent method and overnight hybridization. The signal intensity was lessened compared with chemiluminescent detection but clearly visible. For chromogenic detection, incubation of the hybridized array with substrates re-

\section{ITS2 Mismatches ITS2 Mismatches}

ITS2

ITS4

ITS5

ITS4

ITS5

ITS4

ITS5

ITS4

A

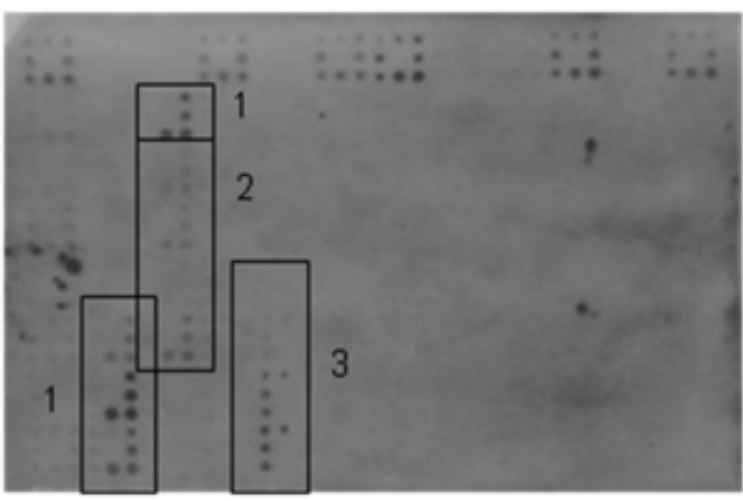

B

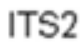

ITS4

ITS5

ITS4

ITS5

ITS4

ITS5

ITS4

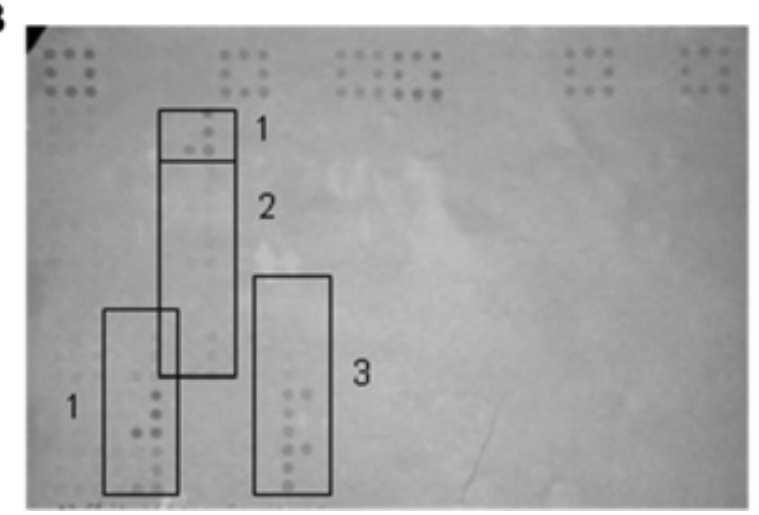

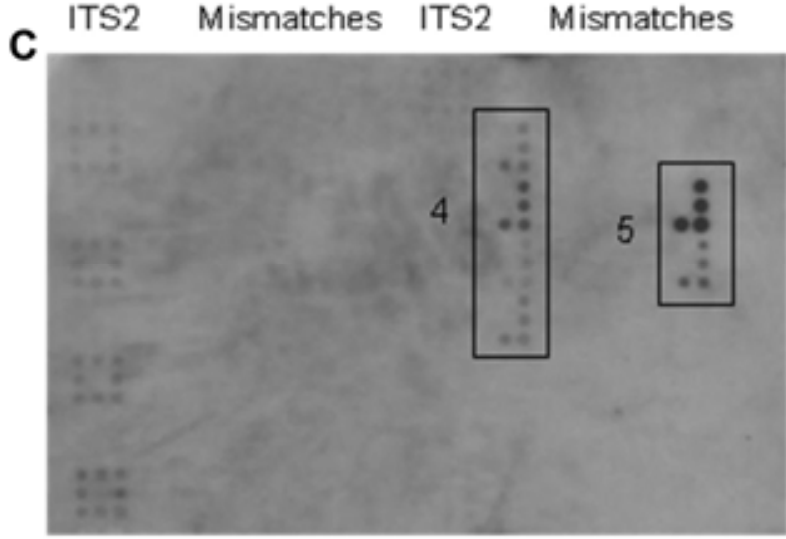

D

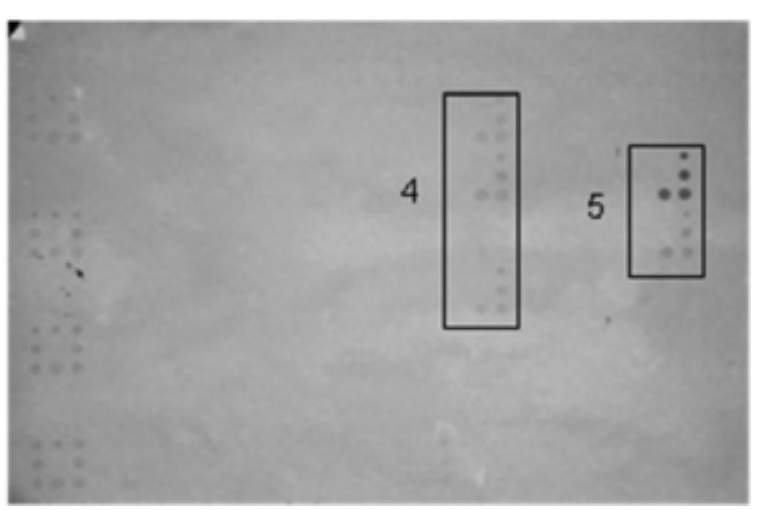

Fig. 4. DNA macroarray detection of solanaceous vegetable pathogens. The array was spotted with $30 \mathrm{pmol}$ of species-specific detector oligonucleotides, hybridized with targets at $10 \mathrm{ng} / \mathrm{ml}$ of hybridization buffer, and incubated overnight at $55^{\circ} \mathrm{C}$. Representative soil sample from high tunnels planted with cherry tomato (A and B) and artificially inoculated pepper with Phytophthora capsici (C and D) using chemiluminescent (A and $\mathbf{C}$ ) and chromogenic (B and D) detection methods. 1, Pyrenochaeta lycopercisi; 2, Phoma destructive; 3, Fusarium oxysporum; 4, Phytophthora capsici; and 5, Solanaceae. Top row and left column include positive and internal controls. Positive controls: internal transcribed spacer (ITS)2, ITS4, and ITS5; internal controls (mismatches): ITS2 C-G central mismatch (ITS2_1_1), ITS2 T-A central mismatch (ITS2_1_2), ITS G-C end mismatch (ITS2_1_3), ITS2 CT-GA central mismatch (ITS2_2_1), and ITS2 GC-CG end mismatch (ITS2_2_2) (17). 
quires more time, at least 2 to $3 \mathrm{~h}$ depending on the abundance of the particular targets in the samples, compared with $1 \mathrm{~h}$ in chemiluminescent detection. Similarly, for environmental samples, detection using the chromogenic method requires overnight incubation with substrates to obtain similar results to chemiluminescent detection. For optimal results, at least 10 pmol of species-specific oligonucleotides in the array and labeled targets of at least $10 \mathrm{ng} / \mathrm{ml}$ of hybridization buffer were required. Lievens et al. (7) also recommended that hybridization of targets at $10 \mathrm{ng} / \mathrm{ml}$ of hybridization buffer should be an appropriate concentration when pure cultures need to be identified.

With both of the detection methods used in this study, signal intensity produced from hybridization varied among the oligonucleotides of each species and depended on the sequence of oligonucleotide used. For example, species-specific oligonucleotides for $S$. solani, Sts4, produced the most intense signals compared with Sts1, Sts2, and Sts3. Also, for P. capsici, signals detected from Pc2, Pc3, and Pc6 were much more intense than those detected from Pc4. This phenomenon also occurred in a study using DNA macroarray to detect vascular wilt pathogens Verticillium alboatrum, V. dahliae, and other tomato pathogens (6). The authors reported that certain oligonucleotides are easily saturated, even at low DNA concentrations.

The entire DNA macroarray detection system could be divided into three components: (i) PCR amplification and target labeling, (ii) hybridization and washing, and (iii) detection and visualization. This study provides information on additional options for each component. These options provide flexibility to users when materials for one component may not be available. For example, one could choose to conduct PCR amplification, target labeling, hybridization, and washing using the DIG labeling or AlkPhos Direct labeling system, and detection and visualization using a chemiluminescent or chromogenic method.

Chromogenic detection was shown to yield similar results compared with chemiluminescent detection in regard to signal specificity, duration of hybridization between the array and targets, and cost. Though the method requires 1 to $2 \mathrm{~h}$ longer for the visualization process, it nevertheless provides a convenient alternative for researchers who lack darkroom facilities but wish to conduct plant disease diagnosis in their own laboratories.

\section{Acknowledgments}

M.-Y. Wong thanks Universiti Putra Malaysia for providing a scholarship to complete a visiting scholar program at the Department of Plant Pathology and Plant-Microbe Biology, Cornell University. This project was supported by the National Research Initiative Competitive Grants Program Grant number 200955605-05184 from the National Institute of Food and Agriculture granted to C. D. Smart.

\section{Literature Cited}

1. Agindotan, B., and Perry, K. L. 2007. Macroarray detection of plant RNA viruses using randomly primed and amplified complementary DNAs from infected plants. Phytopathology 97:119-127.

2. Call, D. R., Brockman, F. J., and Chandler, D. P. 2001. Detecting and genotyping Escherichia coli O157:H7 using multiplexed PCR and nucleic acid microarrays. Int. J. Food Microbiol. 67:71-80.

3. Fessehaie, A., De Boer, S. H., and Levesque, C. A. 2003. An oligonucleotide array for the identification and differentiation of bacteria pathogenic on potato. Phytopathology 93:262-269.

4. Kawasaki, E., Saiki, R., and Erlich, H. 1993. Genetic-analysis using polymerase chain reaction-amplified DNA and immobilized oligonucleotide probes-reverse dot-blot typing. Methods Enzymol. 218:369-381.

5. Lievens, B., Brouwer, M., Vanachter, A. C. R. C., Levesque, C. A., Cammue, B. P. A., and Thomma, B. P. H. J. 2003. Design and development of a DNA array for rapid detection and identification of multiple tomato vascular wilt pathogens. FEMS Microbiol. Lett. 223:113-122.

6. Lievens, B., Brouwer, M., Vanachter, A. C. R. C., Levesque, C. A., Cammue, B. P. A., and Thomma, B. P. H. J. 2005. Quantitative assessment of phytopathogenic fungi in various substrates using a DNA macroarry. Environ. Microbiol. 7:1698-1710.

7. Lievens, B., Claes, L., Vanachter, A. C. R. C., Cammue, B. P. A., and Thomma, B. P. H. J. 2006. Detecting single nucleotide polymorphisms using DNA arrays for plant pathogen diagnosis. FEMS Microbiol. Lett. 255:129-139.

8. Lievens, B., and Thomma, B. P. H. J. 2005. Recent developments in pathogen detection arrays: implications for fungal plant pathogens and use in practice. Phytopathology 95:1374-1380.

9. Lin, C., Chiang, Y., and Tsen, H. 2009. Development and use of a chromogenic macroarray system for the detection of Staphylococcus aureus with enterotoxin A, B, C, D, E, and G genes in food and milk samples. Foodborne Pathogens Dis. 6:445-452.

10. Roche Applied Science, USA. Detection of DIG-labeled Probes. http://www.roche-applied-science.com/sis/lad/index.jsp?id=LA020200

11. Saiki, R. K., Walsh, P. S., Levenson, C. H., and Erlich, H. A. 1989. Geneticanalysis of amplified DNA with immobilized sequence-specific oligonucleotide probes. Proc. Natl. Acad. Sci. USA 86:6230-6234.

12. Sholberg, P., O'Gorman, D., Bedford, K., and Levesque, C. A. 2005. Development of a DNA macroarray for detection and monitoring of economically important apple diseases. Plant Dis. 89:1143-1150.

13. Tambong, J. T., De Cock, A. W. A. M., Tinker, N. A., and Levesque, C. A. 2006. Oligonucleotide array for identification and detection of Pythium species. Appl. Environ. Microbiol. 72:2691-2706.

14. Voordouw, G., Shen, Y., Harrington, C. S., Telang, A. J., Jack, T. R., and Westlake, D. W. S. 1993. Quantitative reverse sample genome probing of microbial communities and its application to oil field production waters. Appl. Environ. Microbiol. 59:4101-4114.

15. White, T. J., Bruns, T., Lee, S., and Taylor, J. 1990. Amplification and direct sequencing of fungal ribosomal RNA genes for Phylogenetics. In: PCR Protocols: A Guide to Methods and Applications. M. A. Innis, D. H. Gelfand, J .J. Sninsky, and T. J. White, eds. Academic Press, New York.

16. Zhang, N., Geisser, D. M., and Smart, C. D. 2007. Macroarray detection of solanaceous plant pathogens in the Fusarium solani species complex. Plant Dis. 91:1612-1620.

17. Zhang, N., McCarthy, M. L., and Smart, C. D. 2008. A macroarray system for the detection of fungal and oomycete pathogens of solanaceous crops. Plant Dis. 92:953-960. 